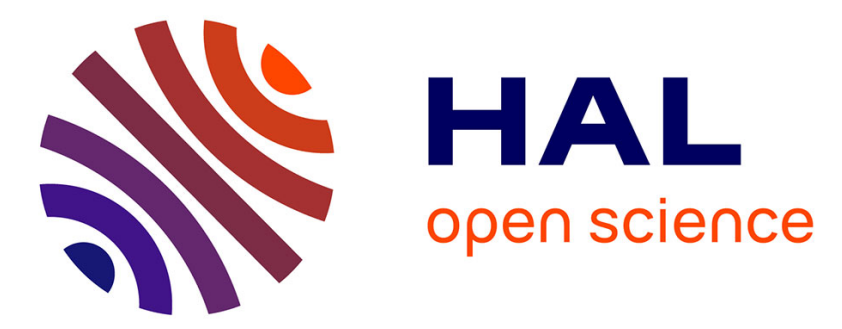

\title{
Regards croisés d'acteurs gérontologiques territorialisés sur la loi Hôpital patients santé et territoires (HPST)
}

Mickaël Blanchet

\section{To cite this version:}

Mickaël Blanchet. Regards croisés d'acteurs gérontologiques territorialisés sur la loi Hôpital patients santé et territoires (HPST). CIST2016 - En quête de territoire(s) ?, Collège international des sciences du territoire (CIST), Mar 2016, Grenoble, France. pp.79-84. hal-01353681

\section{HAL Id: hal-01353681 \\ https://hal.science/hal-01353681}

Submitted on 12 Aug 2016

HAL is a multi-disciplinary open access archive for the deposit and dissemination of scientific research documents, whether they are published or not. The documents may come from teaching and research institutions in France or abroad, or from public or private research centers.
L'archive ouverte pluridisciplinaire HAL, est destinée au dépôt et à la diffusion de documents scientifiques de niveau recherche, publiés ou non, émanant des établissements d'enseignement et de recherche français ou étrangers, des laboratoires publics ou privés. 


\title{
Regards croisés d'acteurs gérontologiques territorialisés sur la loi Hôpital patients santé et territoires (HPST)
}

\author{
AUTEUR \\ Mickaël BLANCHET, REIACTIS (France)
}

\section{RÉSUMÉ}

Issues de la loi relative à l'hôpital, aux patients, à la santé et aux territoires (HPST) du 21 juillet 2009, les agences régionales de santé (ARS) ont pour mission d'assurer, au niveau régional, un pilotage unifié du système de santé. L'objectif de cette agence est de renforcer l'efficience du système de santé et de développer une approche globale et cohérente du parcours de soins. Cette mise en place questionne les équilibres territoriaux et sectoriels qui prévalaient jusque-là. Deux années après la mise en place des ARS, des investigations gérontologiques et géographiques dans trois régions françaises (projet Mapéru) ont pu appréhender les changements et résistances à l'œuvre provoqués par leur création. II ressort de l'observation des actions des ARS et des entretiens menés auprès d'acteurs gérontologiques institutionnels (ARS, conseils généraux) une régionalisation des politiques de santé et un développement des politiques gérontologiques sous un registre économique. Dans ce jeu, le territoire se révèle être davantage un outil politique et économique qu'un espace où se formulent des réponses collectives entre institutions, professionnels et usagers.

\section{MOTS CLÉS}

Territoire, santé, État, territorialisation, efficience

\begin{abstract}
The Regional Agency for Health (ARS) related to the Hospital, Patient, Health and Territories (HPST) Law of 21th of July 2009 are commissionned to, at a regional scale, unify health policies on territories, to strengthen the health system efficiency and to develop a global approach of care. Two years after their creation, the Mapéru project came to observe in three French regions (Bretagne, Pays-de-la-Loire and Rhône-Alpes) the adjustements and the resilience of the gerontological dimension related to this law. According to interviews led with institutional and professionnal actors, the HPST Law encouraged economic norms where territories are used as political and economical supports rather than local spaces where collective solutions are drawn up between institutions, professionals and users.
\end{abstract}

\section{KEYWORDS}

Territory, Health, State, Territorialisation, Efficiency

\section{INTRODUCTION}

Publiée au Journal officiel du 22 juillet 2009, la loi n²009-879 du 21 juillet 2009 portant réforme de l'hôpital et relative aux patients, à la santé et aux territoires comprend quatre titres consacrés respectivement à la modernisation des établissements de santé, à l'accès aux soins, aux mesures de santé publique et à la prévention et, enfin, à l'organisation territoriale du système de santé. Les agences régionales de santé (ARS) sont chargées de coordonner régionalement l'ensemble de ces quatre axes d'action. 
Seulement, la fusion des activités médico-sociales et des activités sanitaires au sein des ARS bouscule les équilibres entre ces mêmes secteurs qui prévalaient jusqu'en 2011.

- Tout d'abord, sur le plan sectoriel, la loi a organisé la fusion des anciennes agences régionales d'hospitalisation et des anciennes directions régionales et départementales des affaires sanitaires et sociales. Cette fusion laisse craindre pour les fonctionnaires de ces institutions et les professionnels médico-sociaux une concentration des moyens et des politiques sur l'hôpital et un gel des anciennes sectorisations.

- Dans le même temps, la contractualisation est l'outil choisi pour faire ce lien : la loi HPST généralise le mécanisme contractuel à l'ensemble des acteurs, du haut en bas de la chaîne de responsabilité. De ce fait, l'État rédige un contrat d'objectifs et de moyens avec chaque agence régionale de santé, qui conclut à son tour un contrat d'objectifs et de moyens avec chaque établissement de santé, mais aussi avec tout opérateur local. Or, qui dit contractualisation dit capacité à contractualiser, donc capacité à s'engager. La loi HPST a donc renforcé les pouvoirs des opérateurs selon des objectifs financiers.

- Sur le versant de l'offre, la création des ARS s'accompagne d'une méthodologie concurrentielle d'appel à projets. Cette concurrence est organisée par les ARS et s'inspire largement des méthodologies des marchés publics. Les ARS s'appuient sur le marché pour réguler l'offre sur le territoire à partir de Contrats pluriannuels d'objectifs et de moyens (CPOM), dans lesquels l'accent est mis sur l'adaptation aux besoins sanitaires des individus et sur l'efficacité économique.

- Enfin, au niveau exécutif, des questions demeurent sur la gouvernance (Tabuteau, 2010) régionale et locale. L'alignement gestionnaire de l'État et des directions hospitalières (Pierru, 2012) ainsi que le passage d'une politique de co-construction avec les collectivités locales à une logique de contractualisation interrogent sur les rapports avec les professionnels et les collectivités locales.

Les fondements de loi HPST sont largement critiqués (Eynaud, 2009) : le laisser-faire en matière de programmation, les objectifs économiques, l'hospitalo-centrisme ainsi que le rôle de faire-valoir des collectivités territoriales en matière de gouvernance refroidissent une partie conséquente des professionnels sanitaires et médico-sociaux. Plus largement, cette réforme s'inscrit dans l'évolution, plus large, des modes de gestion économiques de l'action publique depuis une trentaine d'années en France (Rosanvallon, 1995). Dans cette dynamique, le territoire représente de plus en plus un support d'action destiné à accompagner des populations spécifiques mal prises en compte dans les formes d'intervention antérieures, et ce, sous un modèle contractualiste. Témoin de ce mouvement, l'objectif de cette communication est d'observer, à partir de la notion de territorialisation et sous un angle gérontologique, les changements et résistances à l'œuvre dans le domaine des politiques de la vieillesse provoqués par la loi Hôpital patients santé et territoires (HPST) et la création des ARS. La territorialisation désigne ici le processus complexe d'interaction entre des politiques publiques qui s'adaptent aux spécificités territoriales (démographiques, géographiques, organisationnelles) et le milieu géographique déjà marqué par des inégalités, des pratiques et des héritages distinctifs (Séchet et Fleuret, 2006 ; Curtis et Riva, 2010) ${ }^{1}$. II découle de cette posture théorique plusieurs interrogations. La première porte sur le degré de prise en compte du territoire comme

1 En recentrant son intervention sur des territoires précis, l'action publique a donné au projet local une place déterminante. Elle a également dessiné une géographie prioritaire, en révélant une multitude de territoires d'intervention, sur lesquels étaient mobilisés à la fois les moyens publics et les acteurs locaux, autour de projets clairement identifiés. 
construit social historique auquel concourent les politiques publiques. La seconde porte sur les conséquences, tant au niveau de l'action que des représentations, du repositionnement de l'État dans le domaine gérontologique. Ainsi, comment les nouvelles compétences gérontologiques et médico-sociales de l'État sont elles appréhendées et utilisées par les agences régionales de santé ? Comment ces nouvelles marges de manœuvre territoriales sont-elles utilisées et perçues par les acteurs gérontologiques, départements et professionnels locaux en tête?

\section{MÉTHODOLOGIE}

Pour répondre à ces interrogations, la communication revient sur les conséquences territoriales et gérontologiques de la loi HPST dans trois régions françaises étudiées de mai 2012 à février 2014 dans le cadre d'un post-doctorat relatif à l'accompagnement de la maladie d'Alzheimer en milieu rural ${ }^{2}$. En effet, l'une des parties de ce projet de recherche s'est attachée à observer le déploiement du plan Alzheimer 2008-2012 dans le cadre de la mise en place de trois ARS (Bretagne, Pays-de-laLoire et Rhône-Alpes). Dans cette optique, le financement et la création sous l'égide des ARS de nouveaux dispositifs de coordination professionnel comme les maisons pour l'autonomie et l'intégration des personnes Alzheimer (MAIA), le déploiement de places spécialisées dans les établissements médico-sociaux (pôles d'activités et de soins adaptés - PASA, unités cognitivo-comportementales, accueils de jour ou de nuit en maison de retraites médicalisées et équipes spécialisées Alzheimer au domicile intégrant une ergothérapeute et une psychomotricienne) constituaient une occasion intéressante d'interroger les acteurs gérontologiques sur les conséquences de la loi HPST. Pour rendre compte des perceptions, des rapports et des productions qui découle de cette loi, les recherches ont fait le choix d'interroger les acteurs en distinguant deux niveaux : le niveau institutionnel, où l'on retrouve les fonctions de programmation, planification et financement sur le territoire de l'action gérontologique, et le niveau local où se joue le recours local des personnes âgées à l'offre de services et établissements.

Au niveau institutionnel, les recherches se sont basées sur de nombreuses sources documentaires, sur la conduite de treize entretiens semi-directifs auprès des services des trois agences régionales de santé et des cinq départements de l'étude mobilisés sur le développement de l'action médico-sociale et gérontologique sur le territoire. Deux axes d'interrogation structuraient la conduite de ces treize entretiens. Le premier axe s'intéressait aux représentations qu'avaient les institutions gérontologiques de la loi HPST et des premières actions gérontologiques territorialisées des ARS. Le second axe avait pour but de mesurer les conséquences de ce changement de cadre sur les rapports entres les institutions gérontologiques territorialisées, ainsi que sur le rapport de ces mêmes institutions à leurs territoires d'action, aux professionnels locaux et aux besoins gérontologiques.

Au niveau des deux territoires ruraux d'étude, vingt-sept entretiens semi-directifs auprès d'acteurs médicaux et médico-sociaux ont été réalisés. Les entretiens portaient sur leurs représentations de la loi HPST et la mise en œuvre des politiques des ARS, et les conséquences de ce changement politique sur l'accompagnement gérontologique local.

2 Le projet Mapéru : prise en charge de la maladie d'Alzheimer dans les périphéries rurales des départements à dominante urbaine. 
Que ce soit au niveau local ou institutionnel, les entretiens ont été analysés selon les représentations des acteurs et les faits. Cette analyse a ensuite été croisée à la lecture des documents de planification des conseils généraux, des plans sanitaires et sectoriels des ARS, ainsi que d'autres sources documentaires et statistiques.

\section{ANALYSE DES RÉSULTATS}

II ressort de la reformulation de la gouvernance des politiques de la santé et de la vieillesse plusieurs conséquences que les investigations ont pu mettre en évidence. En premier lieu, cette reformulation politique et territoriale a favorisé l'émergence de plusieurs formes de politiques de santé régionalisées. Ces politiques territorialisées oscillent entre découpages départementaux et infra-départementaux et résultent de compromis sectoriels notamment entre les activités hospitalières et les activités médicosociales. Il découle de ces choix une hétérogénéité des maillages de santé entre les régions (Coldefy et Lucas-Gabrielli, 2012). Dans ce jeu, la réaffirmation des prérogatives de l'État sur le champ médico-social est vécue pour les départements d'étude comme une perte de leadership sur le plan gérontologique. Néanmoins, au-delà de ces rééquilibrages, la loi HPST et la création des ARS actent surtout une orientation de fond des politiques sanitaires et sociales, en faveur des prestations individualisés et de l'efficacité économique.

Tout d'abord cette création entérine le choix de l'individu comme niveau d'action. Si cet effort en direction de l'individu favorise les prises en charge, il questionne les acteurs interrogés sur ses conséquences sociales, politiques et territoriales. Ainsi, la volonté des pouvoirs publics d'élargir le panier de prestations en direction des individus dans un cadre de plus en plus concurrentiel est perçu par les acteurs comme le passage d'une logique d'usage à une logique de consommation (Blanchet, 2011). En effet, l'individualisation des prestations des politiques gérontologiques n'est envisagée que dans une optique économique (Del Volgo et Gori, 2010). Au niveau des pratiques, cette approche économique se concrétise sous deux formes. En premier lieu par une vision managériale des personnes prises en charge. Ainsi, sur le plan gérontologique, la création des MAIA (outil de coordination professionnel) ou bien des PASA (places spécialisées Alzheimer en maison de retraite médicalisée) s'est accompagnée de la volonté « d'homogénéiser » le langage et les pratiques professionnelles et d'optimiser les flux de patients à travers l'introduction de nouveaux concepts managériaux comme la file active (optimisation de la demande selon la capacité d'action) ou la table tactique (assure la mise en œuvre et l'analyse du service rendu). Dans ce cadre, l'adaptation des politiques aux besoins des individus a favorisé la spécialisation de l'offre existante et le développement de nouveaux outils. Néanmoins, ce développement s'opère sous un registre sectoriel voire marketing reléguant ainsi les problématiques de fond qui renvoient invariablement aux questions de professionnalisation, de moyens et de transversalité de l'action.

Selon les discours et les premières actions des ARS de l'étude, la territorialisation constitue autant un processus de formulation de réponses sur le territoire entre des besoins différenciés qu'une opportunité d'ajustement économique. Dans ce sens, l'utilisation excessive du terme " efficience » dans les documents de planification des $\mathrm{ARS}^{3}$ révèle

3 Terme utilisé 23, 14 et 22 fois dans les plans stratégiques régionaux de santé des ARS RhôneAlpes, Pays de la Loire et Bretagne. 
la primauté des programmations économiques. L'observation des premières actions médico-sociales et gérontologiques des ARS de l'étude montre que ces dernières utilisent la concurrence et la contractualisation comme méthodes de régulation. Cette culture du faire au profit du faire-faire, est de plus en plus imitée sur le plan gérontologique par les collectivités territoriales et atteste de la diffusion de normes du privé à des secteurs anciennement cogérés par l'État, les collectivités locales et les professionnels (Simard et Lascoumes, 2011). L'imposition de ces normes concurrentielles se traduit par une territorialisation concurrentielle et administrative de l'action médico-sociale et gérontologique qui modifie les anciens modes locaux de prise en charge. Selon les représentations des institutions et des acteurs locaux médico-sociaux, elle contribue à standardiser l'action médico-sociale selon le degré d'efficacité économique et, par ricochet, elle alimente la distance entre les financeurs gérontologiques, ARS en tête, et les professionnels locaux qui accompagnent quotidiennement les personnes âgées. Dans ce sens, il en découle un nouveau rapport de ces politiques médico-sociales au territoire, ce dernier étant perçu dans les territoires ruraux d'étude comme un moyen d'ajustement social et économique. Cette perception très forte dans ces territoires masque pourtant les possibilités offertes par le levier contractuel de la loi HPST, celui de faire du territoire un moyen souple et pertinent de construction de politiques médico-sociales et gérontologiques locales. L'élargissement de cette étude dans le temps, mais aussi à d'autres territoires et à de nouveaux interlocuteurs (élus, caisses de retraites notamment), contribuera à enrichir les connaissances sur la dimension géographique des politiques médicales et sociales de la vieillesse.

Ces conclusions seront alimentées de septembre 2015 à février 2016 par des contacts réguliers avec les acteurs de l'étude (conseils départementaux, ARS, acteurs gérontologiques locaux...) qui permettront de compléter les résultats exposés.

\section{RÉFÉRENCES}

Blanchet M., 2011, Politiques de la vieillesse dans la région Pays de la Loire, thèse de géographie sous la direction de Ch. Pihet, Angers.

Coldefy M., Lucas-Gabrielli V., 2012, « Le territoire, un outil d'organisation des soins et des politiques de santé ? Évolution de 2003 à 2011 », Questions d'économie de la santé, IRDES, n 175, pp. 1-8.

Curtis S., Riva M., 2010, "Health geographies II: complexity and health care systems and policy", Progress in Human Geography, n 34, pp. 513-520.

Del Vogo M.-J., Gori R., 2012, « Résister à la société de la norme et de l'évaluation », Connexions, n 94, pp. 49-60.

Eynaud M., 2009, « Contribution à la réflexion sur la réforme de l'hôpltal : à propos des limites de la loi HPST », Informations psychiatriques, 85(2), pp. 117-121.

Pierru F., 2012, « Les recompositions paradoxales de l'État sanitaire français, Transnationalisation, étatisation et individualisation des politiques de santé ", Éducation et sociétés, $\mathrm{n}^{\circ}$ 30, pp. 107-129.

Rosanvallon P., 1995, La nouvelle question sociale. Repenser l'État-providence, Paris, Le Seuil.

Séchet R., Fleuret S., 2006, "Spatialité des enjeux de pouvoir et des inégalités : pour une géographie sociale de la santé ", in Séchet R., Veschambre V. (dir.), Penser et faire la géographie sociale. Contributions à une épistémologie de la géographie sociale, Rennes, PUR, 332-349.

Simard L., Lascoumes P., 2011, «L'action publique au prisme de ses instruments », Revue française de science politique, $\mathrm{n}^{\circ} 61$, pp. 5-21.

Tabuteau D., 2010, "Loi "Hôpital, patients, santé et territoires" (HPST): des interrogations pour demain !», Santé publique, 1/2010, vol. 22, pp. 78-90 
CIST2016 proceedings

En quête de territoire(s) ? Looking for territories?

\section{L'AUTEUR}

Mickaël Blanchet

Réseau d'études international sur

l'âge, la citoyenneté et l'intégration

socio-économique-REIACTIS

mickaelblanchetfr@gmail.com 